\title{
Application of Polycrystalline SiGe for Gain Control in SiGe Heterojunction Bipolar Transistors
}

\author{
V.D.Kunz, C.H. de Groot, S.Hall ${ }^{\#}$, I.M.Anteney, A.I.Abdul-Rahim, P.Ashburn \\ Department of Electronics \& Computer Science, University of Southampton, Southampton \\ SO17 1BJ, England \\ \# Department of Electrical Engineering \& Electronics, University of Liverpool, Liverpool, L69 \\ $3 B X$, England
}

\begin{abstract}
This paper reports a method of controlling the gain of a bipolar transistor by incorporating $G e$ in the polysilicon emitter. Measured results show that varying the Ge content in the polysiGe from 0 to $33 \%$ gives a change in base current of approximately four. The competing influences of the Ge and the interfacial layer at the polySiGe/Si interface are investigated theoretically using an effective surface recombination velocity for the polySiGe emitter. Good agreement between theory and measured results is obtained. When incorporated into a SiGe HBT, the polySiGe emitter will allow the best trade-off between gain and $B V_{C E O}$ to be achieved for a given $G e$ and $B$ profile in the base.
\end{abstract}

\section{Introduction}

To achieve high values of $\mathrm{f}_{\mathrm{T}}$ and $\mathrm{f}_{\max }$ in SiGe HBT's, it is necessary to minimise delay times in the base and collector of the transistor [1-3]. The base delay is minimised by using a narrow basewidth and large $\mathrm{Ge}$ gradient across the base to create a built-in electric field [1]. Such aggressive Ge profiles can give very high values of common emitter current gain B [3]. The collector delay is minimised by increasing the collector doping to decrease the transit time and to suppress the Kirk effect. Unfortunately high collector doping concentrations have the disadvantage of degrading the common emitter breakdown voltage $B V_{C E O}$ of the transistor [4].

The trade-off between $B V_{C E O}$ and $\beta$ can be understood from the following well known equation:

$$
\mathrm{BV}_{\mathrm{CEO}}=\frac{\mathrm{BV}_{\mathrm{CBO}}}{\sqrt[n]{\beta}}
$$

This equation shows that if the gain of the transistor is too high, the common emitter breakdown voltage is degraded. To address this problem, a method is needed of reducing the gain without modifing the base Ge and B profiles.

In SOI CMOS technology, single-crystal SiGe has been used in the sources of MOS tranistors to supress parasitic bipolar gain[5].

In this paper, $\mathrm{Ge}$ is incorporated in polycrystalline silicon to create a novel polySiGe emitter. This approach is compatible with existing BiCMOS technologies in which a polysilicon emitter is used to make contact to the very shallow emitter/base junction. When applied to a SiGe HBT, this method will allow the gain to be controlled independently of the Ge and B profiles in the base.

An issue that needs to be addresses in polySiGe emitters is the competing influence of the Ge, which gives increased base current, and the interfacial layer, which gives reduced base current. A theory is developed which quantifies these two competing machanisms and a comparison made with measured devices.

\section{Transistor Fabrication}

Silicon bipolar transistors were fabricated with polySiGe emitters with Ge contents in the range 0 to $33 \%$. The in-situ phosphorus doped poly SiGe layer was deposited in a Thermo VG Semicon CV200 LPCVD system at a temperature of $540^{\circ} \mathrm{C}$ after an ex-situ $\mathrm{HF}$ etch. The polySiGe emitter was completed by annealing for $30 \mathrm{~s}$ at either 900 or $800^{\circ} \mathrm{C}$. SIMS measurements were made to characterise the germanium, phosphorus and oxygen profiles in the polySiGe emitter, and Gummel plots were measured to characterise the electrical properties of the resulting bipolar transistors.

\section{Results}

Figure 1 shows a typical SIMS profile for a polySiGe layer with $11 \% \mathrm{Ge}$ after an anneal of $30 \mathrm{~s}$ at $800^{\circ} \mathrm{C}$. It can be seen that the $\mathrm{Ge}$ and phosphorus profiles are reasonably uniform across the polySiGe layer, and there 
is very little penetration of the phosphorus into the single-crystal silicon. The oxygen profile shows a dose of $6.4 \times 10^{14} \mathrm{~cm}^{-2}$ oxygen at the polySiGe/Si interface. Assuming a uniform silicon dioxide layer, this is equivalent to an interfacial oxide thickness of $0.21 \mathrm{~nm}$.

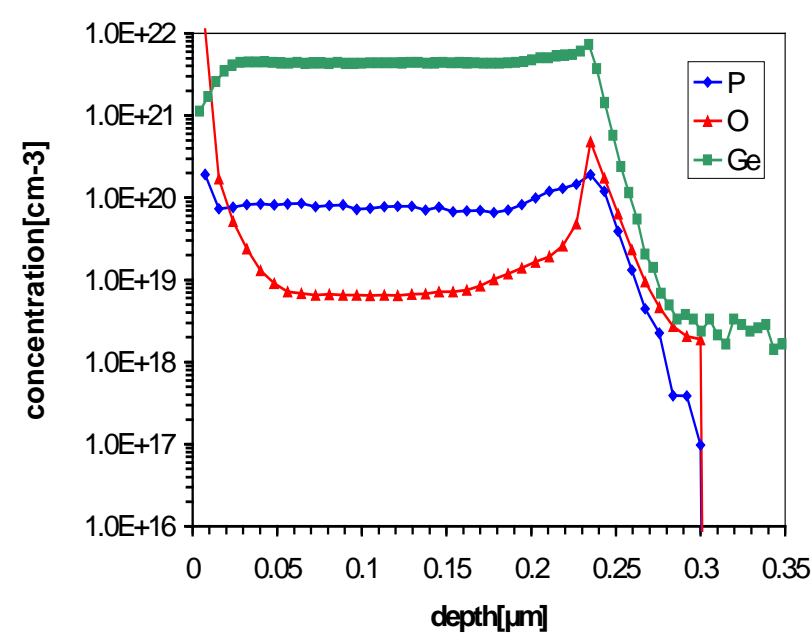

Figure 1: Typical SIMS profile for a polySi ${ }_{0.89} \mathrm{Ge}_{0.11}$ emitter given an anneal of 30 s at $800^{\circ} \mathrm{C}$.

Figure 2 compares Gummel plots for transistors with polySi and polySiGe emitters given an anneal of 30s at $900^{\circ} \mathrm{C}$. The collector characteristics are ideal with ideality factors of 1.07 and the base characteristics nearideal with minimum ideality factors of 1.20, 1.14 and 1.13 at $\mathrm{V}_{\mathrm{BE}}=0.6 \mathrm{~V}$ for 0,10 and $19 \% \mathrm{Ge}$ respectively. The base current increases with Ge content, rising by a factor of 2.9 on going from 0 to $10 \%$ and by a factor of 4.0 on going from 0 to $19 \%$.

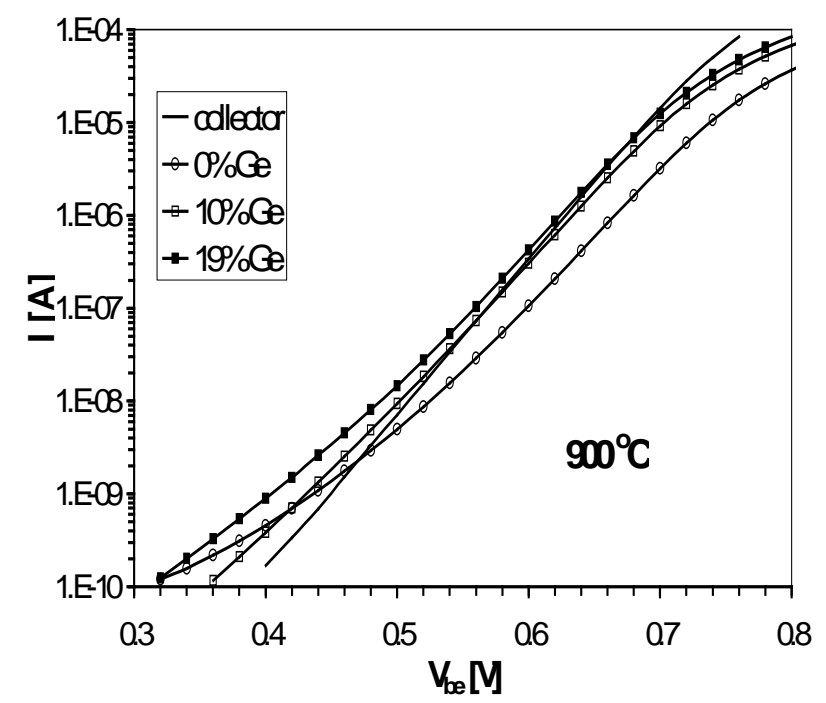

Figure 2: Gummel plots of transistors with polySi and polySiGe emitters annealed for $30 \mathrm{~s}$ at $900^{\circ} \mathrm{C}$.

Figure 3 shows similar Gummel plots for transistors with polySi and polySiGe emitters annealed for 30s at $800^{\circ} \mathrm{C}$. The base characteristics of these transistors are less ideal than those in figure 2 , with minimum ideality factors of $1.3,1.3$ and 1.2 at $\mathrm{V}_{\mathrm{BE}}=0.55 \mathrm{~V}$ for 0,11 and $33 \%$ Ge respectively. This is due to the very light anneal used in the transistor fabrication and the consequent small degree of phosphorus penetration into the singlecrystal silicon, as shown in figure 1 . Nevertheless, these Gummel plots show a similar trend to those in figure 2, namely an increase in base current with increasing $\mathrm{Ge}$ content. This increase is a factor of 3.3 on going from 0 to $11 \%$ Ge and 4.0 on going from 0 to $33 \%$.

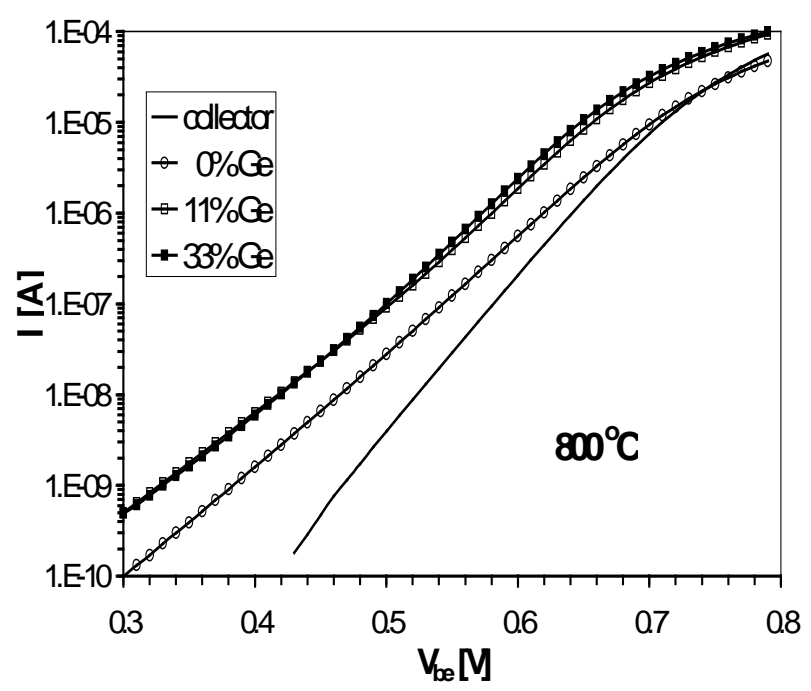

Figure 3: Gummel plots of transistors with polySi and polySiGe emitters annealed for $30 \mathrm{~s}$ at $800^{\circ} \mathrm{C}$.

\section{Theory}

In order to explain the above dependence of base current on Ge content in the polySiGe emitter, a theory has been developed for polySiGe emitters. The approach used defines an effective surface recombination velocity for the polySiGe emitter analogous to that used by $\mathrm{Yu}$ et al [6] for polySi emitters.
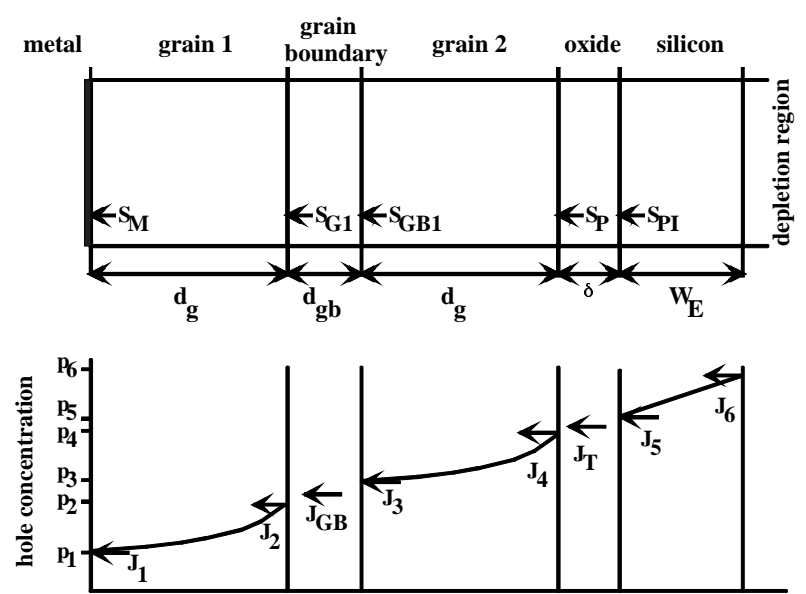

Figure 5: Schematic illustration showing the definition of effective surface recombination velocities. 
An effective surface recombination velocity is defined at each interface in the polySiGe emitter, as illustrated in figure 5. The current at each interface is then written in terms of these recombination velocities. For example, the current density $J_{l}$ adjacent to the metal contact is given by:

$$
\mathrm{J}_{1}=\mathrm{qS}_{\mathrm{M}} \mathrm{p}_{1 \mathrm{SiGe}}
$$

where $S_{M}$ is the surface recombination velocity at the metal contact and $p_{\text {ISiGe }}$ is the hole concentration in the SiGe layer adjacent to the metal contact.

Following the work of $\mathrm{Yu}$ et al [6], an equation for the current density $J_{4}$ at the left of the interfacial oxide layer can be defined:

$$
\mathrm{J}_{4}=\mathrm{qS}_{\mathrm{P}} \mathrm{p}_{4 \mathrm{SiGe}}
$$

where $S_{P}$ is an effective surface recombination velocity given by:

$$
\mathrm{S}_{\mathrm{P}}=\mathrm{a}_{\mathrm{g}}-\frac{\mathrm{b}_{\mathrm{g}}^{2}}{\mathrm{a}_{\mathrm{g}}+\mathrm{S}_{\mathrm{GB} 1}}
$$

The parameters $a_{g}$ and $b_{g}$ depend on the physical properties of the polySiGe grains and are given by:

$$
\mathrm{a}_{\mathrm{g}}=\frac{\mathrm{D}_{\mathrm{pSiGe}}}{\mathrm{L}_{\mathrm{pSiGe}}} \operatorname{coth}\left(\frac{\mathrm{d}_{\mathrm{g}}}{\mathrm{L}_{\mathrm{pSiGe}}}\right)
$$

and

$$
\mathrm{b}_{\mathrm{g}}=\frac{\mathrm{D}_{\mathrm{pSiGe}}}{\mathrm{L}_{\mathrm{pSiGe}}} \operatorname{csch}\left(\frac{\mathrm{d}_{\mathrm{g}}}{\mathrm{L}_{\mathrm{pSiGe}}}\right)
$$

where $d_{g}$ is the grain width, and $L_{p S i G e}$ and $D_{p S i G e}$ are the hole diffusion length and hole diffusivity in the polySiGe grain. The recombination velocity after the grain boundary shown in figure 5 is given by:

$$
\begin{aligned}
& \mathrm{S}_{\mathrm{GB} 1}=\mathrm{a}_{\mathrm{gb}}-\frac{\mathrm{b}_{\mathrm{gb}}^{2}}{\mathrm{a}_{\mathrm{gb}}+\mathrm{S}_{\mathrm{G} 1}} \\
& \mathrm{a}_{\mathrm{gb}}=\frac{\mathrm{D}_{\mathrm{gbSiGe}}}{\mathrm{d}_{\mathrm{gb}}}+\mathrm{S}_{\mathrm{gbSiGe}} \\
& \mathrm{b}_{\mathrm{gb}}=\frac{\mathrm{D}_{\mathrm{gbSiGe}}}{\mathrm{d}_{\mathrm{gb}}}
\end{aligned}
$$

where $d_{g b}$ is the grain boundary width, and $D_{g b S i G e}$ is the hole diffusivity in the polySiGe grain boundary and $S_{g b S i G e}$ the recombination velocity at the grain boundary interface. $S_{G I}$ is then given by:

$$
\mathrm{S}_{\mathrm{G} 1}=\mathrm{a}_{\mathrm{g}}-\frac{\mathrm{b}_{\mathrm{g}}^{2}}{\mathrm{a}_{\mathrm{g}}+\mathrm{S}_{\mathrm{M}}}
$$

Transport across the interfacial oxide layer is assumed to be by tunnelling, and the band diagram is illustrated in figure 6 .

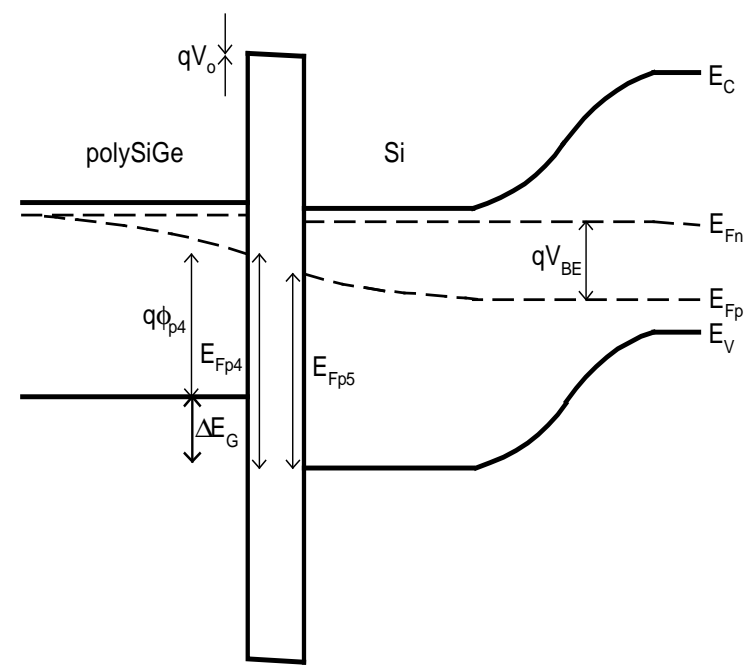

Figure 6: Band diagram of a polySiGe emitter

Using this band diagram, and noting that the energy $E_{F p 4}$ contains the bandgap difference between $\mathrm{Si}$ and SiGe $\Delta E_{G}$, the following equation for the effective surface recombination velocity $S_{P I}$ of the polySiGe emitter can be derived:

$$
S_{P I}=S_{I}+\left(\frac{T_{I}\left(S_{P}+S_{I}\right)}{\frac{T_{I}}{F}+S_{P}+S_{I}}\right)
$$

where the parameter $F$ represents the effects of the Ge:

$$
\mathrm{F}=\frac{\mathrm{N}_{\mathrm{VSiGe}}}{\mathrm{N}_{\mathrm{VSi}}} \exp \left(\frac{\Delta \mathrm{E}_{\mathrm{G}}}{\mathrm{kT}}\right)
$$

In these equations $T_{I}$ models tunnelling through the interfacial layer and $S_{I}$ recombination at the polySiGe/Si interface.

\section{Discussion}

In practical polySiGe emitters, there will be a conflict between increased base current from the presence of the $\mathrm{Ge}$ and decreased base current from the interfacial layer. To illustrate this interaction, figure 7 shows a graph of the modelled value of $S_{P I}$ as a function of interfacial layer thickness. In calculating the value of $S_{P I}$, the parameter values in [6] were used. At high values of interfacial layer thickness, $T_{I}$ is small and hence $S_{P I} \approx S_{I}$ in equation 11. Consequently, the $\mathrm{Ge}$ in the polySiGe layer 
has no effect on the value of $S_{P I}$. At low values of interfacial layer thickness, $T_{I}$ in equation 11 is large, so $S_{P I}$ approaches a value of $F\left(S_{P}+S_{I}\right) \approx F S_{P}$. In this situation the polySiGe layer has a strong effect on the value of $S_{P I}$.

Using the measured value of interfacial oxide thickness obtained from the SIMS profile in figure 1 $(0.21 \mathrm{~nm})$, figure 7 predicts an increase in $S_{P I}$ by a factor of 3.4 on going from $0 \%$ to $10 \% \mathrm{Ge}$ and 4.8 on going from $0 \%$ to $19 \% \mathrm{Ge}$. These factors are consistent with the measured increases in base current seen in figure 2, namely 2.9 and 4.0. In a shallow polySiGe emitter, where there is little recombination in the single-crystal emitter, it would be expected that the base current would be proportional to $S_{P I}$. In which case, the above comparison demonstrates good agreement between theory and measurement. Higher increases in the value of $S_{P I}$ could be obtained by using a thinner interfacial layer. For example, an interfacial layer thickness of $0.1 \mathrm{~nm}$ would give an increase in $S_{P I}$ by a factor of 13.5 for $20 \%$ Ge.

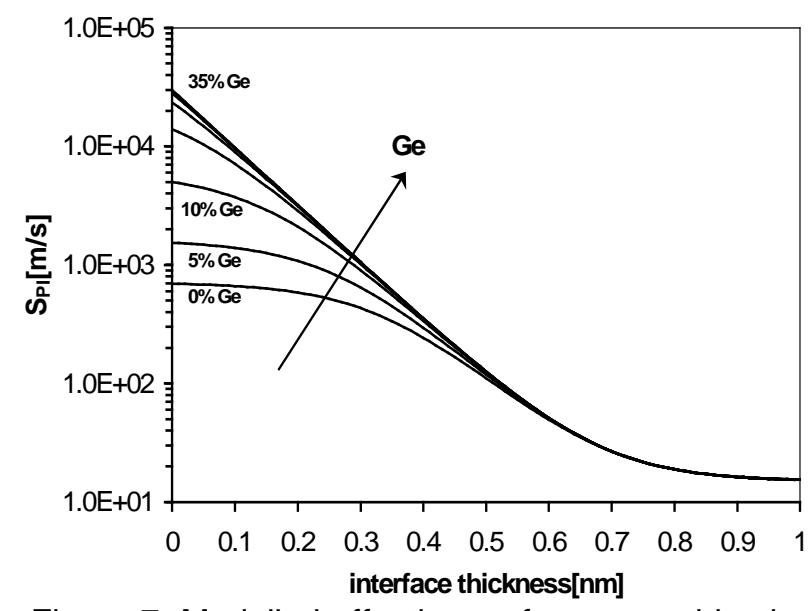

Figure 7: Modelled effective surface recombination velocity $S_{P I}$ as a function of interfacial layer thickness for Ge contents of 0 to $35 \%$ in $5 \%$ steps.

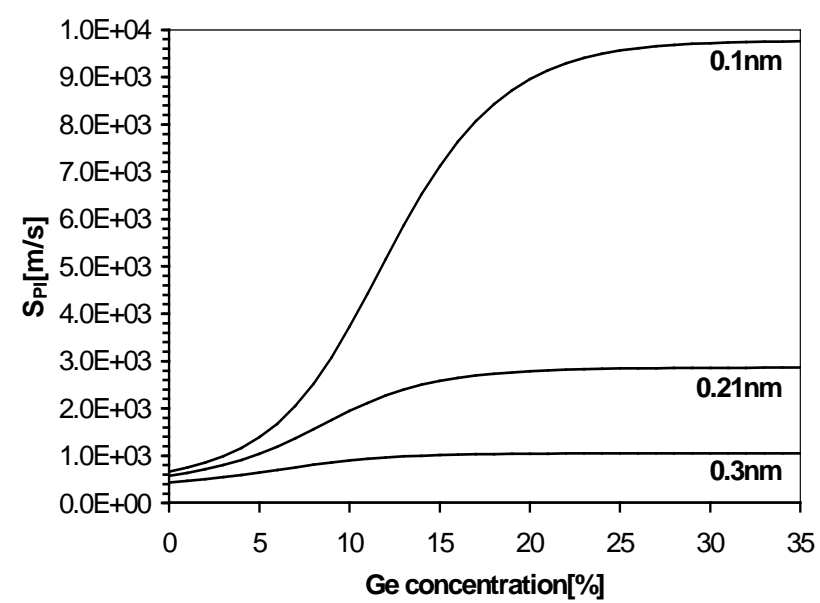

Figure 8: Modelled effective surface recombination velocity $S_{P I}$ as a function of Ge contents for different interfacial layer thickness
The increase in the value of $\mathrm{S}_{\mathrm{PI}}$ achieved by $\mathrm{Ge}$ incorporation in the polySi emitter is very rapid at low $\mathrm{Ge}$ concentrations, but saturates at high $\mathrm{Ge}$ concentrations. This is illustrated in figure 8 for thin interfacial oxide layers. For an interfacial oxide thickness of $0.1 \mathrm{~nm}, S_{P I}$ saturates for Ge concentrations greater than approximately $20 \%$. This situation occurs because the term $T_{I} / F$ in equation 11 becomes smaller than $\left(\mathrm{S}_{\mathrm{P}}+\mathrm{S}_{\mathrm{I}}\right)$ as the $\mathrm{Ge}$ content rises. With increasing interfacial layer thickness, the onset of saturation occurs at lower Ge concentrations. In practice there is therefore little benefit to be obtained by increasing the Ge concentration above $20 \%$.

\section{Conclusions}

It has been shown that the incorporation of Ge into a polySi emitter allows the base current to be adjusted independently of the base profile. Measured results show that Ge content of $10 \%$ gives an increase in base current by a factor of 4 . Theory predicts that higher factors could be achieved by reducing the interfacial layer thickness.

\section{Acknowledgement}

This work was funded by EPSRC and the European Commission.

\section{References}

[1] G.Freeman, D.Ahlgren, D.R.Greenberg, R.Groves, F.Huang, G.Hugo, B.Jagannathan, S.J.Jeng, J.Johnson, K.Schonenberg, K.Stein, R.Volant, S.Subbanna; "A $0.18 \mu \mathrm{m}$ 90GHz f $\mathrm{f}_{\mathrm{T}} \mathrm{SiGe}$ HBT BiCMOS, ASIC-compatible, copper interconnect technology for $\mathrm{rf}$ and microwave applications"; IEDM Technical Digest (1999)

[2] J.Böck, T.F.Meister, H.Knapp,, D.Zöschg, H.Schäfer, K.Aufinger, M.Wurzer, S.Boguth, M.Franosch, R.Stengl, R.Schreiter, M.Rest, L.Treitinger; "SiGe bipolar technology for mixed digital and analogue rf applications"; IEDM Technical Digest, pp745-748 (2000)

[3] K.Washio, M.Kondo, E.Ohue, K.Oda, R.Hayami, M.Tanabe, H.Shimamoto, T.Harade; "A $0.2 \mu \mathrm{m}$ self-aligned selective epitaxial growth $\mathrm{SiGe} \mathrm{HBT}$ featuring $107 \mathrm{GHz} \mathrm{f}_{\max }$ and 6.7ps ECL"; IEEE Transactions on Electron Devices, vol 48, pp1989-1994 (2001)

[4] B.Martinet, H.Baudry, O.Kermarrec, Y.Campidelli, M.Laurens, M.Marty, T.Schwartzmann, A.Monroy, D.Bensahel, A.Chantre; "100GHz SiGe:C HBTs using nonselective epitaxy"; Proc. European Solid State Device Research Conf. pp97-100 (2001)

[5] J.-h. Sim, C.H.Choi, K.Kim; "Elimination of parasitic bipolar-induced breakdown effects in ultra-thin SOI MOSFETs using narrow-bangap-sources (NBS) structure"; IEEE Transactions on Electron Devices, vol 42, pp1495-1502 (1995) [6] Z.Yu, B.Ricco, R.W.Dutton; IEEE Trans. Electron Devices, vol 31, p773 (1984) 